\title{
The Madura Foot: A case of eumycotic mycetoma on histopathology
}

\author{
Jadhav D. $\mathrm{S}^{1}$, Paul A. $\mathrm{U}^{2}$, Baste B. $\mathrm{D}^{3}$, Valand A. $\mathrm{G}^{4}$ \\ ${ }^{1}$ Dr. D. S. Jadhav, Associate Professor, ${ }^{2}$ Dr. Anuja U. Paul, Resident, ${ }^{3}$ Dr. B. D. Baste, Assistant Professor, ${ }^{4}$ Dr. A. G. \\ Valand, Professor and Head Department of Pathology, S.R.T.R. GMC. Ambajogai, Maharashtra, India.
}

Address for Correspondence: Dr. D. S. Jadhav, Email: drdsjjadhav@rediffmail.com

\begin{abstract}
Madura foot is a deep mycosis commonly seen in tropical and subtropical countries such as India. It is caused by two groups of organisms, bacteria belonging to the group of Actinomycetes and the true mycetes named eumycetes. The incidence is more common in agricultural workers. Generally these lesions were present on foot \& shows presence of induration, fibrosis and minimal discharge from sinuses. Though culture remains the gold standard diagnostic test histopathology plays important role in the early diagnosis and definitive treatment of these cases.
\end{abstract}

Keywords: Madura foot, Eumycetoma, Actinomycetoma

\section{Introduction}

Mycetoma is a chronic granulomatous disease of skin, subcutaneous tissue and bones that is present worldwide and is endemic in tropical and subtropical regions. Commonly affected site by this infection is the foot and was described by Gill in Indian for the first time in Madura district in 1842, hence the name Madura Foot $[1,2]$. It is a slow growing infection presenting with characteristic symptomatic triad of swelling, draining sinuses and extrusion of colonial grains in the exudates. As the disease has slow and relatively pain free progression, it is usually diagnosed at an advanced stage $[2,3]$. The most common site of occurrence is the foot $(70 \%$ cases $)$, explaining the synonym 'Madura foot' [4]. Mycetoma is commonly seen in agricultural workers and in barefoot walkers in dry and dusty areas. Repeated trauma or implantation by thorns and splinters provide a portal of entry for the organism. Infection can be caused by true fungi (eumycetoma) in $40 \%$ cases and by filamentous bacteria (actinomycetes) in $60 \%$ cases [5]. Since the treatment of these two etiologies is entirely different, a definite diagnosis after histopathological and microbiological examination is mandatory [2]. We present a case of Madura foot, diagnosed on histopathology.

\section{Case Report}

A 50 years old male, agricultural worker, admitted in hospital with indurated swelling along with discharging sinuses over foot for the last 1 to 2 years. The swelling was progressively enlarging and associated with pain while walking. Clinical examination revealed a tumefaction, draining sinus discharging purulent exudates. Complete hemogram, Erythrocyte sedimentation rate, C- reactive protein were within normal limits. Tests for Syphilis, HIV infection, Hepatitis $\mathrm{B}$ and $\mathrm{C}$ virus infection were negative. Clinical diagnosis was Madura foot. A deeper biopsy from the lesion was sent for histopathological examination. We received skin covered tissue piece of size 10X9X2 having ulcer of size $3 \times 2$ discharging pus [Fig.1]. On cut section multiple abscess cavities were seen [Fig 2]. Repeated fungal cultures were negative. Histopathological examination showed dense infiltration by acute inflammatory cells in the form of microabscesses along with pale eosinophillic necrotic material with abundant lymphocytes, plasma cells, multinucleated giant cells and abundant septate, branching fungal hyphae (Fig3) Gram stain was negative. Periodic acid Schiff (PAS) stains provided excellent contrast and delineated $4-5 \mu \mathrm{m}$ thick septate hyphae of eumycetoma [Fig.4,5]. A diagnosis of Eumycotic mycetoma was made and the patient was treated with antifungals. There was a good clinical response.

Manuscript received: $16^{\text {th }}$ July 2017

Reviewed: $26^{\text {th }}$ July 2017

Author Corrected: $7^{\text {th }}$ August 2017

Accepted for Publication: $14^{\text {th }}$ August 2017 


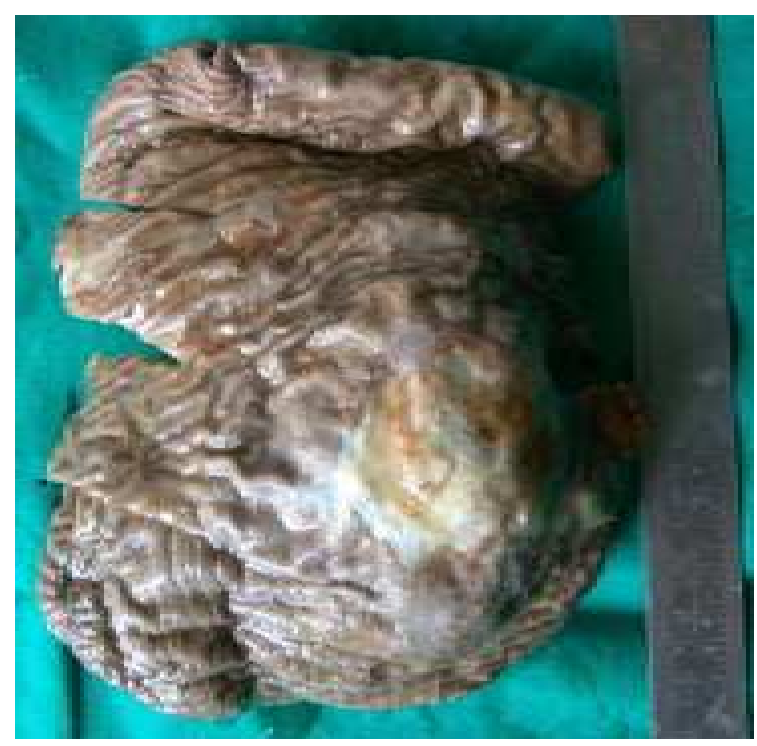

Fig-1: Indurated skin with central ulceration surrounded by granulation tissue

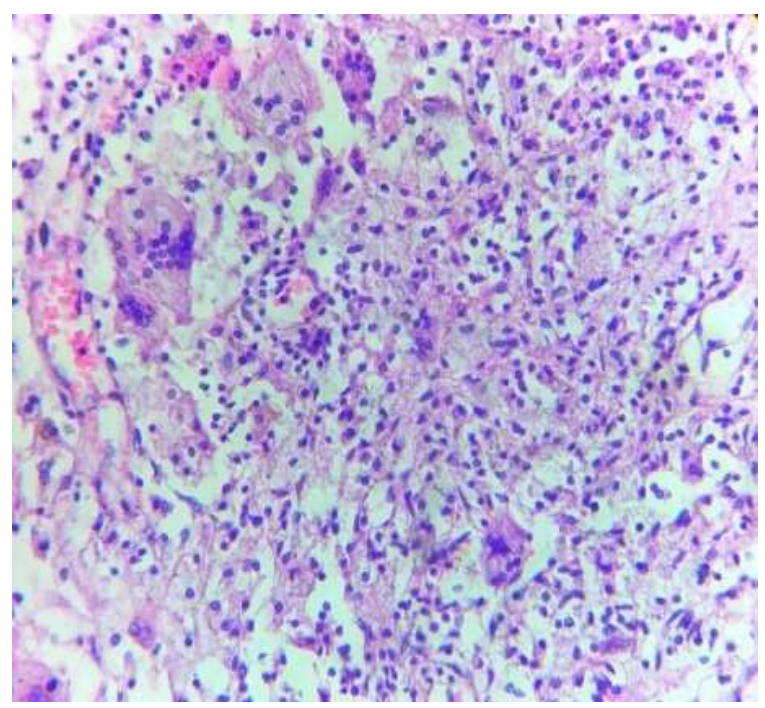

Fig-3: $\mathrm{H}$ and $\mathrm{E}$ stained section shows inflammatory cells and multinucleated giant cells [H\&E: 40X]

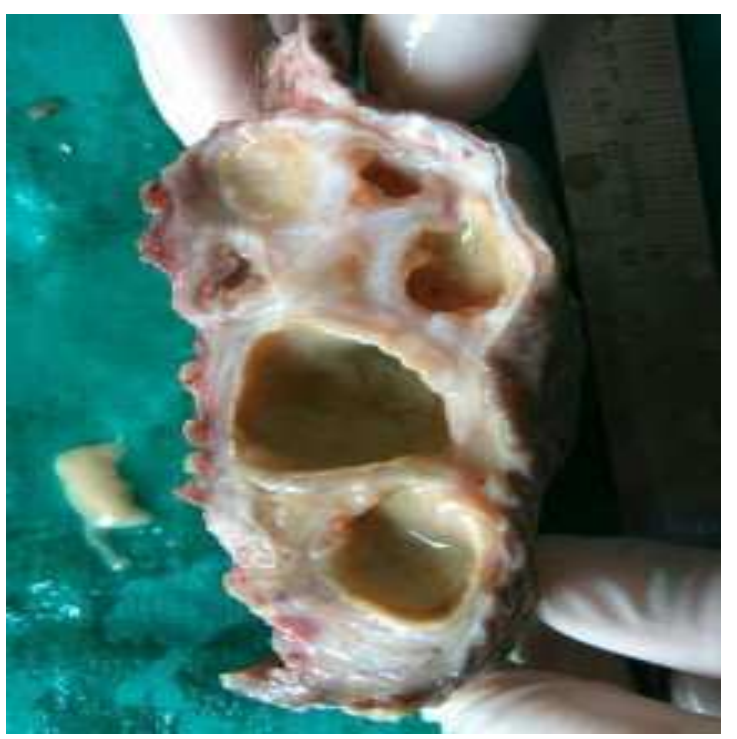

Fig-2: C/S shows multiloculated subcutaneous abscess

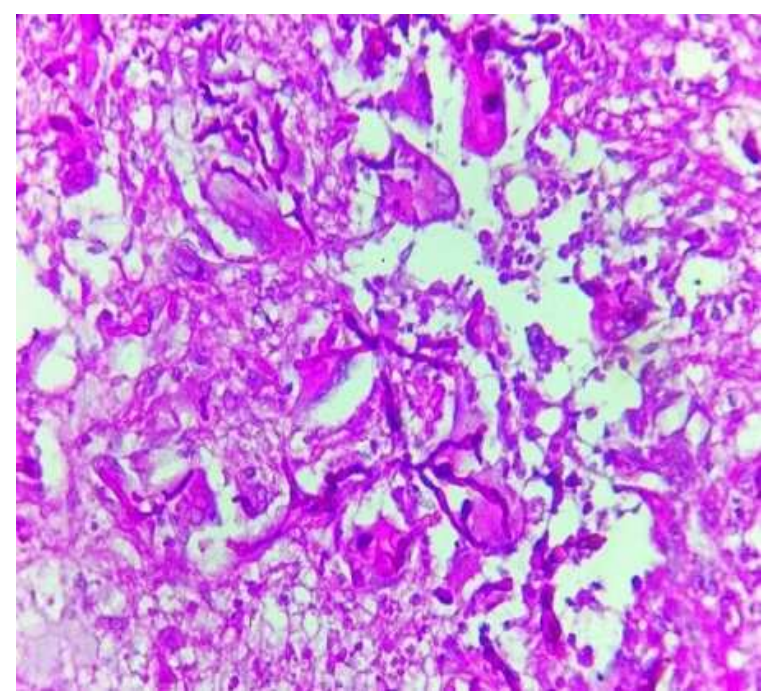

Fig-4: PAS stained section shows delineated septate hyphae of Eumycetes [PAS: 40X]

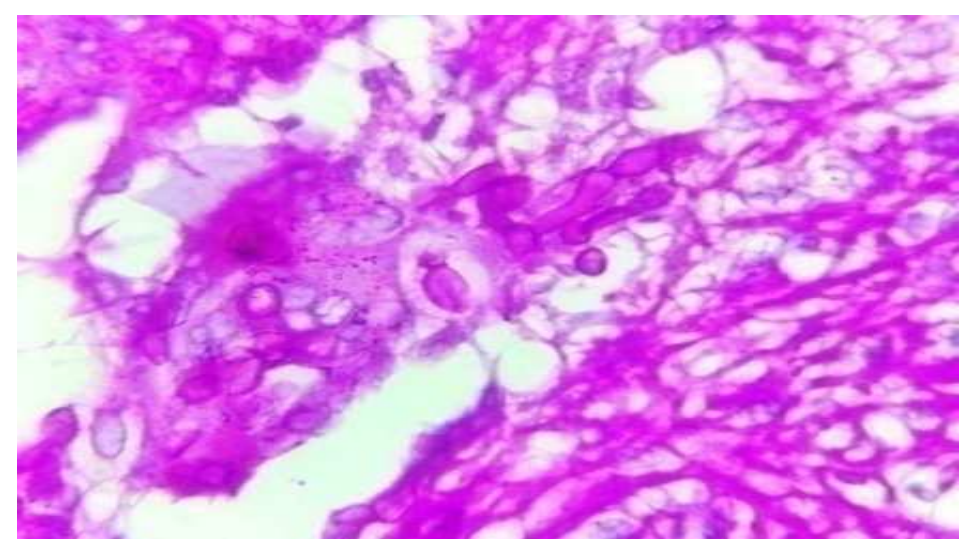

Fig-5: PAS stained section shows delineated septate hyphae of Eumycetes [PAS: 100X] 


\section{Discussion}

Mycetoma is mainly found in dry tropics and affects agricultural workers and people who walk barefooted [6]. Fungi are found as saprophytes in the soil and are introduced through skin wounds. Infection begins in the skin and subcutaneous tissue causing local papular or nodular swelling which grows and ruptures forming discharging sinus tracts exuding characteristic coloured grains [7]. The granules vary in size, colour and consistency depends on the etiological species. These grains are the hallmark of mycetoma [6]. Some sinuses heal with scarring with simultaneous appearance of fresh sinuses in the proximal areas. Subsequently bone destruction occurs [7]. The causative agents have been identified as: the actinomycetes group of bacteria and true mycetes i.e. eumycetes [1]. Over 30 species have been identified to cause mycetoma $[5,8]$.

Actinomycotic mycetoma is caused by aerobic species of actinomycetes belonging to the genera Nocardia, Streptomyces and Actinomadura. Eumycotic mycetoma is associated with a variety of fungi, the most common being Madurella mycetomatis, Pseudoallescheria boydii and Acremonium species [6]. The grains discharged from the sinuses vary in size, colour and consistency. The characteristic features of the grains in combination with the clinical picture (indurated swelling of the foot with multiple discharging sinuses) can be used for rapid provisional identification of the etiological agent [5]. The size of the grains varies from microscopic to 1$2 \mathrm{~mm}$ in diameter. Large grains are seen with madurellae, Actinomadura madurae and A. palletieri whereas granules of $\mathrm{N}$. brasiliences, $\mathrm{N}$. cavae and $\mathrm{N}$. asteroids are small [9]. Dark (black) grains are found only among the eumycotic mycetoma [10]. The incubation period varies from several weeks to months [5]. Sinuses develop after 6-12 months and extension to involve the underlying fascia, muscle and bone is common. In eumycotic mycetoma, there may be multiple punched out lytic lesions in bones. Actinomycotic mycetoma is characterized by both osteolytic and osteosclerotic lesions [6].

Thin filaments of actinomycetoma and thick filaments of eumycetoma can also be differentiated on discharged granules crushed on a slide and stained with lactophenol blue stain [2]. Histopathological examination proves useful in differentiating actinomycetoma from eumycetoma. In cases of Madura foot, biopsy material stained with Haematoxylin and Eosin shows grains or colonies with or without surrounding granulomatous reaction. Eumycotic colonies are frequently surrounded by fibrotic tissue [11]. A Gram stain is of considerable value in distinguishing between actinomycetoma and eumycetoma. The granules of actinomycetoma consisting of fine, branching filaments, only about 1 um thick are gram positive whereas the grains of eumycetoma are gram negative [9]. Eumycotic grains are better identified by PAS and GMS stains and are composed of 4-5 $\mu \mathrm{m}$ thick septate hyphae [12]. In cases of PAS- positive eumycotic colonies showing hyphae, one should look for amorphous matrix highlighted by Gram's stain or PAS stain.

The presence of an amorphous matrix narrows the diagnosis to three eumycotic agents, Madurella mycetomatis, Madurella grisea and Leptosphaeria. If this amorphous matrix is present throughout the colony imparting a grainy appearance, a provisional diagnosis of M. mycetomatis can be considered [11]. Confirmation of diagnosis and exact identification of the species requires culture. Culture, however, is difficult practically and may be false negative many a times [7]. Serodiagnosis with ELISA also is not always diagnostic because there are variable levels of humoral response to infection [4]. In addition, ancillary investigations such as PCR are not readily available at all centres [11]. Thus histology has a beneficial role and remains the only option in culture negative cases. Imaging studies are useful in defining the extent of disease [5]. Besides Mycetoma, the clinical differential diagnoses in patients presenting with chronic discharging sinuses in extremities include Tuberculous osteomyelitis, Blastomycosis, Coccidioidomycosis, Sporotrichosis, Botryomycosis, Syphilis, Yaws and Neoplastic pathologies [5]. The choice of treatment for mycetoma depends on the causative agent which has been identified on the basis of morphology of grain in histopathology sections.

Actinomycetoma can be treated with surgical debridement including prolonged antibiotic treatment for several months. However, resistant cases can be treated with a combination of Trimethoprim sulphomethoxazole, dapsone and streptomycin along with rifampicin. Eumycetomas are only partially responsive to anti- fungal therapy but can be treated by 


\section{Case Report}

surgery due to their normally well circumscribed nature .Surgery in combination with azole treatment is the recommended regime for small eumycetoma lesions in extremities [2]. Amputation is indicated in advanced mycetoma with severe secondary bacterial infections $[4,13]$, not responding to medical treatment; emphasizing the importance of early and definite diagnosis [14].

\section{Conclusion}

Due to slow and relatively pain free progression of the disease, mycetoma is often at an advanced stage when first diagnosed. Prognostically, actinomycetoma can be cured with surgical debridement and appropriate antibiotic therapy while eumycetoma is only partially responsive to antifungal agents, has high rate of recurrence and may require amputation. For mycetomas in which causative infectious agents cannot be isolated, histology may prove beneficial by avoiding inadvertent use of combined antifungal and antimicrobial agents so that a correct therapeutic modality can be decided.

Funding: Nil, Conflict of interest: None initiated, Permission from IRB: Yes

\section{References}

1. Azzoni R, Capitza P; Madura's foot in native of the Philippines immigrant in northern Italy.J Orthop., 2005; 2 (6): 1-6.

2. Alam K, Maheshwari V, Bhargava S, Jain A, Fatima U, Haq EU. Histological diagnosis of madura foot (mycetoma): a must for definitive treatment. J Glob Infect Dis. 2009 Jan; 1(1):64-7. doi: 10.4103/0974777X. 52985.

3. Davis JD, Stone PA, McGarry JJ. Recurrent mycetoma of the foot. J Foot Ankle Surg. 1999 JanFeb; 38(1):55-60.

4. Fahal AH. Mycetoma: a thorn in the flesh. Trans R Soc Trop Med Hyg. 2004 Jan;98(1):3-11.
5. Magana M. Mycetoma. Int J Dermatol. 1984 May; 23(4):221-36.

6. Iffat H, Abid K. Mycetoma Revisited. NDermatol Online, 2011; 2(3): 147-150.

7. Mohammad N, Arif C, Ruksana P, Rokon U,Abdur R, Moydul H; The Madura foot. A CaseReport. N Dermatol Online, 2011; 2(2): 70-73.

8. Negroni R, Lopez Daneri G, Arechavala A,Bianchi MH, Robles AM; Clinical and microbiological study of mycetomas at theMuniz Hospital of Buenos Aires between 1989 and 2004. Rev Argent Microbiol., 2006; 38 (1):13-18.

9. Pilsczek FH, Augenbraun M. Mycetoma fungal infection: multiple organisms as colonizers or pathogens? Rev Soc Bras Med Trop. 2007 Jul-Aug; 40 (4): 463-5.

10. van de Sande WW, de Kat J, Coppens J, Ahmed AO, Fahal A, Verbrugh H, van Belkum A. Melanin biosynthesis in Madurella mycetomatis and its effect on susceptibility to itraconazole and ketoconazole. Microbes Infect. 2007 Jul; 9 (9):1114-23. Epub 2007 May 18.

11. Chufal SS, Thapliyal NC, Gupta MK. An approach to histology-based diagnosis and treatment of Madura foot. J Infect Dev Ctries. 2012 Sep 17;6(9):684-8. doi: 10.3855 / jidc. 2387 .

12. Taralakshmi VV, Pankajalakshmi VV, Arumugam S, Subramanian S. Mycetoma caused by Madurella mycetomii in Madras. Australas J Dermatol. 1978 Dec; 19 (3):125-9.

13. Fahal AH, Mycetoma: Clinico-pathological Monograph, University of Khartoum Press. 2006, pp 23-30

14. Ahmed Hassan Fahal; Mycetoma. Khartoum Medical Journal, 2011; 4(1): 514 - 523.

\section{How to cite this article?}

Jadhav D. S, Paul A. U, Baste B. D, Valand A. G. The Madura Foot: A case of eumycotic mycetoma on histopathology. Trop J Path Micro 2017;3(3):309-312.doi: 10.17511/jopm.2017.i3.14. 